\title{
The prevalence of secretor status and co-expression of lewis antigen in voluntary blood donors
}

\author{
R Raj Bharath 1 , P Arumugam² \\ ${ }^{1}$ Assistant Professor, ${ }^{2}$ Professor, Department of Transfusion Medicine, The Tamil Nadu Dr. MGR Medical Univesity, \\ Chennai, Tamil Nadu, India
}

\section{A B S T R A C T}

Background: Blood group substances are present in soluble form in a majority of individuals in secretion such as saliva and body fluids. Secretor status refers to the presence (SeSe and Sese) or absence (sese) of secretor gene which secrete $A B H$ soluble substances. Secretor status can be used to resolve $A B O$ discrepancies of people whose blood group cannot be identified by routine blood grouping and it can also help in identifying patients who may be a high risk group for getting certain diseases. Aims and Objectives: Our aim and objectives of the study is to find out the Prevalence of Secretor Status and Co-expression of Lewis Antigens among the Voluntary Blood Donors. Materials and Methods: This study was conducted in sixty volunteers and the method used to determine the secretor status was hemagglutination inhibition method. Their blood was used to detect the type of Lewis (Le) antigen since the type of Lewis antigen correlated with the secretor status of the individual. Results: Among the sixty subjects tested, forty five of them were found to be secretors and fifteen of them were Non-secretors. The number of Lewis $(a+b-)$ individuals were twelve, Lewis $(a-b+)$ were thirty nine and Lewis (a-b-) were nine. Conclusion: The prevalence of secretors was $75 \%$ and non-secretors were $25 \%$ respectively. We found $65 \%$ of the volunteers were found to be Le $(a-b+)$ positive, $20 \%$ were Le $(a+b-)$ and the remaining $15 \%$ were Le (a-b-) which correlated with the $\mathrm{ABH}$ antigen secretor status.

Access this article online

Website:

http://nepjol.info/index.php/AJMS

DOI: 10.3126/ajms.v7i5.14848

E-ISSN: 2091-0576

P-ISSN: $2467-9100$

Key words: Secretors, Non-secretors, Lewis antigen, Hemagglutination inhibition

\section{INTRODUCTION}

Blood group substances are present in soluble form in a majority of individuals in secretion such as saliva and body fluids. Secretor status refers to the presence (SeSe and Sese) or absence (sese) of secretor gene which secrete $\mathrm{ABH}$ antigen soluble substances. ${ }^{1}$ The biosynthesis of $\mathrm{H}$ antigen found on red blood cells and that found in secretions involves two different $\alpha, 1,2$ - fucosyl transferase (FUT) enzymes encoded by two closely linked genes on chromosome 19, FUT-1 and FUT-2. These genes are also referred to as the $\mathrm{H}$ gene and $\mathrm{Se}$ (secretor) gene. Both encode enzymes that add on $\mathrm{H}$ specific fucose sugar to a precursor oligosaccharide core structure but they act on different precursor substances. The terminal carbohydrate sequences of the $\mathrm{ABH}$ antigens in saliva and plasma are identical to those on red cells. But the backbone or the framework carbohydrate structures are different.

$\mathrm{H}$ antigen is synthesized in RBCs when the H (FUT 1) gene encoded fucosyl transferase attaches a fucose via an $\alpha 1,2$ linkage to the terminal galactose of type 2 precursor chains. $\mathrm{H}$ antigen in secretions is synthesized when the Se gene encoded fucosyl transferase attaches a fucose via an $\alpha 1,2$ linkage to the terminal galactose of type 1 precursor chains in secretory tissues. ${ }^{2}$

The ability to secrete $\mathrm{ABH}$ antigens is genetically inherited, approximately $80 \%$ are secretors and $20 \%$ are nonsecretors. ${ }^{3}$ This percentage of secretor and non secretor 
varies according to the race and ethnicity. This trait is inherited as a single locus gene in simple mendelian fashion. The secretor gene is dominant and non secretor is recessive. People having the secretor gene are called Secretors and those who do not have are called Non-secretors. ${ }^{3}$ In secretor individuals of the appropriate $\mathrm{ABO}$ group, $\mathrm{ABH}$ antigens are detected in the secretions of the goblet cells and mucous glands of the digestive (saliva, gastric juice, bile, meconium), genitourinary (spermatic fluid, vaginal secretion, ovarian cyst fluid, urine) and respiratory tracts as well as in other secretions (milk, sweat, tears and amniotic fluid). ${ }^{4}$

The ABO, Lewis (Le) and Secretor/Non secretor system are considered an entity in which all antigens are chemically related but in which independent system of genes determine the phenotype. ${ }^{5}$ The Lewis gene (FUT 3) resides on Chromosome 19 and is distantly linked to $\mathrm{H}$ and Se loci. Lewis antigens, unlike antigens of all other blood group system are not intrinsic to the RBC but are synthesized in intestinal epithelial cells. Lewis antigens circulate in plasma while bound to glycosphingolipids-and are passively adsorbed onto RBCs. The presence of the particular type of Lewis antigen approximately indicates the secretor status of the individual. Individuals who type as Le $(a-b+)$ are secretors, Le $(\mathrm{a}+\mathrm{b}-)$ are nonsecretors and Le (a-b-) individuals could be either secretors or nonsecretors. Lewis system is associated with disease such as peptic ulcers and bladder cancer. Le $\mathrm{e}^{\mathrm{b}}$ is associated with Helicobacter Pylori infection. The incidence of Lewis blood group phenotype in Asian population is $72 \%$ for $\mathrm{Le}^{\mathrm{b}}, 22 \%$ for $\mathrm{Le}^{\mathrm{a}}, 6 \%$ for Le (a-b-) and $3 \%$ for Le (a+b+) individuals. ${ }^{6} \mathrm{~S}$ Akhter et al in his study of blood donors in Dhaka, Bangaldesh reported $19 \%$ were Le (a+b-), $53 \%$ were Le $(a-b+), 26 \%$ were Le (a-b-) and $2 \%$ were Le $(a+b+){ }^{6}$

Sara et al in her results demonstrated that the secretor status plays an intrinsic role in resistance to H.pylori infection and suggested that the fucosylated secretor $\mathrm{ABH}$ antigens constitute interactive members of human and primate mucosal innate immune system.Non secretion of blood group antigen influences the pathogenic sequel of urinary tract infection.?

Camps et al in his study of 1000 alcoholic patients reported that the percentage of non secretors were $32.7 \%$. The frequencies of non secretors varied in different part of British Isles where the study was undertaken. He noted there were a general pattern of increase in group-A non secretors and Le individuals among the alcoholic patients. ${ }^{8}$

Dayaprasad et al in his study of non secretor status - a predisposing factor for vaginal candidiasis had reported that the prevalence of vaginal candidiasis was significantly higher in non secretor group. In his control group, the percentage of secretors and non secretors were $77 \%$ and $23 \%$ respectively. ${ }^{9}$

Genotyping is regarded as the gold standard method in secretor and Lewis blood grouping because it is able to identify minor errors which can occur in agglutinationbased phenotyping..$^{10}$ Our aim and objective of our study was to find out the Prevalence of Secretor Status and Co-expression of Lewis Antigens among the Voluntary Blood Donors

\section{AIM AND OBJECTIVES}

To find out the Prevalence of Secretor Status and Coexpression of Lewis Antigens among the Voluntary Blood Donors

\section{MATERIALS AND METHODS}

This study was conducted in the Department of transfusion Medicine, The Tamilnadu Dr.M.G.R Medical University. A total number of 60 voluntary blood donors donated their blood and participated in this study. Ethical clearance was obtained and informed written consent was obtained from the volunteers who donated blood in the study. The blood groups of the subjects were determined using conventional tube technique. Their blood was tested for the Lewis antigen by using Lewis antisera (Dia Clon Anti- Le ${ }^{a}$ and Anti- Le from Bio rad).

The method used to determine the secretor status was hemagglutination inhibition method. The secretors have water soluble blood group substances which are readily detected in very minute quantities because they have the property of reacting with their corresponding antibodies and thereby neutralizing or inhibiting the capacity of antibody to agglutinate erythrocytes possessing antigen. The reaction provides a means of finding the relative activity or potency of these water soluble blood group substances.

Five $\mathrm{ml}$ of saliva was collected from all voluntary blood donors and it was placed in a boiling water bath to inactivate the salivary enzymes. Antisera are added to the saliva and incubated for 10 minutes at room temperature. To that appropriate pooled cells are added and incubated for 45 minutes at $37^{\circ} \mathrm{C}$. Agglutination of RBC indicates that the person is a non-secretor and its absence indicates the person is a secretor ${ }^{11}$ (Table 1$)$.

Lewis antigen was determined from the blood of all volunteers using the Lewis antisera Le $(\mathrm{a}+\mathrm{b}-)$ and Le $(\mathrm{a}-\mathrm{b}+)$. 
Table 1: Determination of secretor status by hemagglutination inhibition method

\begin{tabular}{llllll}
$\begin{array}{l}\text { Diluted antisera+ } \\
\text { Supernatant saliva }\end{array}$ & $\begin{array}{l}\text { Incubate for } \\
8 \text { to } 10 \mathrm{~min} .\end{array}$ & $\begin{array}{l}\text { Addition of appropriate } \\
\text { indicator red cells }\end{array}$ & $\begin{array}{l}\text { Incubate for } \\
30 \text { to } 60 \text { minutes }\end{array}$ & $\begin{array}{l}\text { Centrifuge } \\
\text { Agglutination }\end{array}$ & $\begin{array}{l}\text { Nonsecretor } \\
\text { No Agglutination }\end{array}$ \\
\hline
\end{tabular}

\begin{tabular}{|c|c|c|c|c|}
\hline & $\begin{array}{l}\text { Lewis } \\
\text { (a+b-) }\end{array}$ & $\begin{array}{l}\text { Lewis } \\
(a-b+)\end{array}$ & $\begin{array}{l}\text { Lewis } \\
\text { (a-b-) }\end{array}$ & Total \\
\hline Secretors & - & 39 & 6 & 45 \\
\hline Nonsecretors & 12 & - & 3 & 15 \\
\hline Total & 12 & 39 & 9 & 60 \\
\hline
\end{tabular}

$\begin{aligned} & \text { Table 3: ABO blood group \& secretor status of } \\
& \text { the participants }\end{aligned}$
\begin{tabular}{lccc}
\hline Blood Group & Secretor & Non secretor & Total \\
\hline A & 9 & 4 & 13 \\
B & 16 & 5 & 21 \\
O & 17 & 6 & 23 \\
AB & 3 & 0 & 3 \\
Total & 45 & 15 & 60 \\
\hline
\end{tabular}

\section{RESULTS}

Among the 60 subjects tested, 45 of them were found to be secretors and 15 of them were Non-secretors. The number of Lewis (a+b-) individuals were 12, Lewis $(\mathrm{a}-\mathrm{b}+)$ were 39 and Lewis (a-b-) were 9.

\section{DISCUSSION}

In our study the prevalence of secretors was $75 \%$ and nonsecretors were $25 \%$ respectively (Figure 1 ). The percentage of secretors and non-secretors varies with the racial and ethnic population. In Caucasians about $20 \%$ of people are non-secretors while our study showed $25 \%$ of our volunteers are non-secretors. ${ }^{12}$

Agarwal in his study of $\mathrm{ABH}$ secretor status in North India found out that $64 \%$ were secretors, $21 \%$ were non secretors and 14\% were aberrant secretors. Individuals who have substance $\mathrm{H}$ in their saliva or those who have substance $\mathrm{H}$ but not $\mathrm{A}$ and $\mathrm{B}$ substances are called as aberrant secretors. ${ }^{13}$

Luiz et al control group were $76.1 \%$ secretors and $23.9 \%$ nonsecretors in his study of patients infected or uninfected by the H.pylori bacillus. ${ }^{14}$

The blood sample of the 60 persons was also tested for the presence of Lewis antigen. In our study, 39 were found to be Le $(a-b+)$ positive, 12 were Le $(a+b-)$ positive and the remaining 9 were Lewis (a-b-) (Figure 2). The 39 Le $(a-b+)$

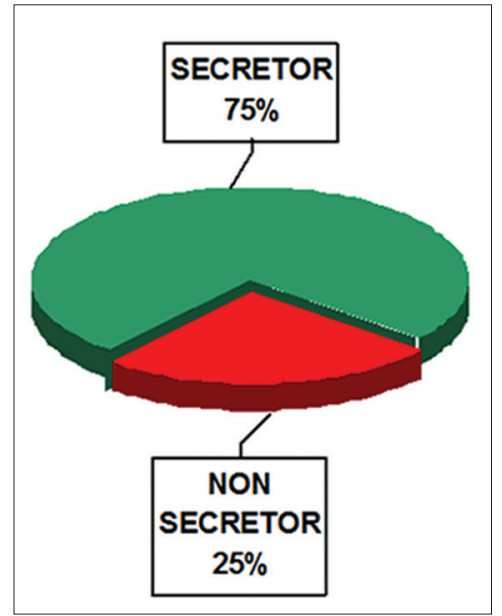

Figure 1: Secretor status

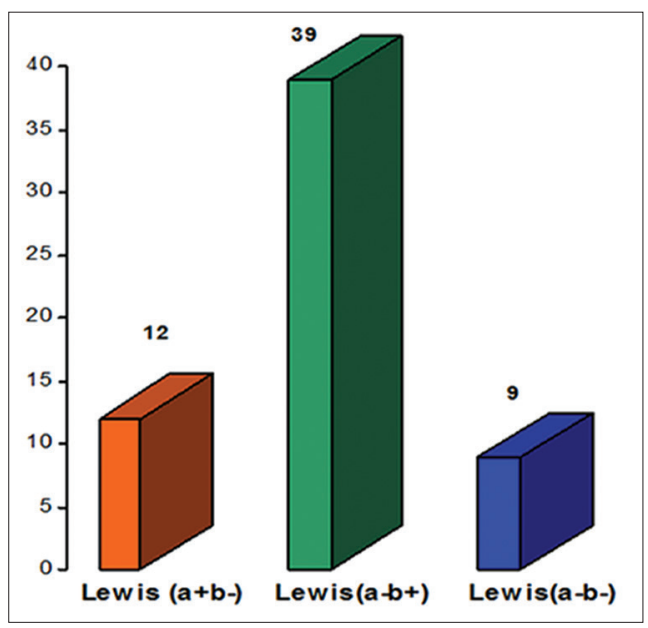

Figure 2: Frequencies of lewis antigens

were secretors and the 12 Le ( $a+b-)$ were nonsecretors. Six of them were secretors and three of them were nonsecretors in the remaining nine Le (a-b-) (Table 2). S Akhter et al in his study of forty two individuals reported $19 \%$ were Le (a+b-), $53 \%$ were Le (a-b+), $26 \%$ were Le (a-b-) and $2 \%$ were Le $(\mathrm{a}+\mathrm{b}+) .{ }^{15}$ Our study revealed A group volunteers had more percentage of non-secretors $(31 \%)$ when compared to B (24\%) and $\mathrm{O}$ groups (26\%) (Table 3).

Tereza et al reported higher percentage of Lewis (a-b-) in black population and suggested that the reason could be due to nongenuine Lewis phenotype (Lewis antigen absent on red cells but present on salivary secretions). She also attributed this high frequency of Lewis (a-b-) phenotype due to decreased concentration of circulating Lewis antigen 
occurring during diseases caused by parasites, infection or other pathologic conditions. ${ }^{16}$ The limitations of the study was the relatively low sample size which cannot be used as an assessment of prevalence of secretor status in the local population.

\section{CONCLUSION}

The prevalence of secretors was $75 \%$ and non-secretors were $25 \%$ respectively. In our study, $65 \%$ of the volunteers were found to be Le (a-b+) positive, $20 \%$ were Le $(a+b-)$ and the remaining 15\% were Le (a-b-) which correlated with the prevalence of $\mathrm{ABH}$ antigen secretor status. Secretor status of the individual can be used to resolve $\mathrm{ABO}$ discrepancies which may occur in certain conditions like Leukemia, Hodgkin disease and Acquired B. It also helps in finding out rare subgroups of $\mathrm{A}$ and $\mathrm{B}$, where normal haemaggulitination method fails. The haemaggulitination inhibition method of finding out the secretor status is cost effective and can be done in any laboratory centre. Co-expression of Lewis antigens on red blood cells plays a contributory role in assessing $\mathrm{ABH}$ secretor status of the individual. However, for the doubtful cases of $\mathrm{ABH}$ Secretors or Lewis antigens expression, the only unambiguous option available is the secretor gene analysis.

\section{REFERENCES}

1. Harmening DM. Modern blood banking and Transfusion practices. Fifth Edition 2008:133.

2. Hillyer CD, Silberstein L, Ness PM, Anderson KC and Roback JD. Blood banking and transfusion Medicine. Second Edition 2007: pp 73-75.

3. Simon TL, Dzik W, Snyder EL, Stowell CP, and Strauss RG. Rossi's Principles of Transfusion Medicine 2002. Third Edition: 97.
4. Daniels G. Human Blood Groups. Second Edition 2002:29.

5. Andresen PH. Relations between the ABO, Secretor/NonSecretor and Lewis Systems with particular reference to the Lewis system. Bisperbjerg Hospital, Copenhagen 1961:396-412.

6. Akhter S, Kibria GM, Akhter NR, Habibulah MM, Islam SMK and Zakariah M. ABO and Lewis blood grouping with $\mathrm{ABH}$ Secretor and Non-secretor status: A cross sectional study in Dhaka. Faridpur Med Coll J 2011; 6(1):38-40.

7. Linden S, Mahdavi J, Semino-Mora C, Olsen C, Carlstedt I, and Boren T. Role of ABO Secretor status in mucosal innate immunity and H.pylori infection. PLoS Pathogens 2008; 4(1): 6-12.

8. Camps FE, Dodd BE and Lincoln PJ. Frequencies of Secretors and Non-secretors of ABH Group Substances among 1000 Alcoholic patients. British Medical Journal 1969; 4: 457-459.

9. Kulkarni DG and Venkatesh D. Non-Secretor Status. A predisposing factor for vaginal candidiasis. Indian $\mathrm{J}$ Physiol Pharmacol 2003; 48(20):225-229.

10. Vestergaard EM, Hein HO, Meyer H, Grunnet N, Jorgensen J, and Torben HW. Reference Values and Biological Variation for Tumor Marker CA 19-9 in Serum for Different Lewis and Secretor Genotypes and Evaluation of Secretor and Lewis Genotyping in a Caucasian Population. Clinical Chemistry 1999; 45: 54-61.

11. Roback JD, Grossman BJ, Harris T, and Hillyer CD. AABB Technical Manual $17^{\text {th }}$ Edition: $883-885$.

12. Harmening DM. Modern Blood Banking and Transfuion Practices. Fifth edition: 150-153.

13. Agarwal $\mathrm{S}$. $\mathrm{ABH}$ Antigens in North Indians. Rev Brazil Genet 1988; 11(1): 165-170.

14. Carlos de Maltos L, Rodrigues J, Eduardo F, de Cassia R, Artur M and Moreria HW. ABO, Lewis, Secretor and Non-secretor phenotypes in patients infected or uninfected by the H.pylori bacillus. Sao Paulo Med J/Rev Paul Med 2002; 20(2):55-58.

15. Akhter S, Kibria GM, Akhter NR, Habibulah MM, Islam SMK, Zakariah $\mathrm{M}$. $\mathrm{ABO}$ and Lewis blood grouping with $\mathrm{ABH}$ secretor and non-secretor status: A cross sectional study in Dhaka. Faridpur Med Coll J 2011;6(1):38-40.

16. Corvelo TCO, Aguiar DCF, and Sagica FES. The expression of $\mathrm{ABH}$ and Lewis antigens in Brazilian semi isolates Black communities. Genetics and Molecular Biology 2002; 25(3): 259-263.

\footnotetext{
Authors Contribution:

RRB - Concept and design of the study, collected data,reviewed the literature, manuscript preparation and critical revision of the manuscript; PA - Manuscript preparation and critical revision of the manuscript.
}

Source of Support: Nil, Conflict of Interest: None declared. 